PROCEEDINGS OF THE

AMERICAN MATHEMATICAL SOCIETY

Volume 138, Number 3, March 2010, Pages 1033-1046

S 0002-9939(09)10139-9

Article electronically published on November 2, 2009

\title{
LINEARLY REPETITIVE DELONE SYSTEMS HAVE A FINITE NUMBER OF NONPERIODIC DELONE SYSTEM FACTORS
}

\author{
MARÍA ISABEL CORTEZ, FABIEN DURAND, AND SAMUEL PETITE \\ (Communicated by Bryna Kra)
}

\begin{abstract}
In this paper we prove linearly repetitive Delone systems have finitely many Delone system factors up to conjugacy. This result is also applicable to linearly repetitive tiling systems.
\end{abstract}

\section{INTRODUCTION}

The concepts of tiling dynamical systems and Delone dynamical systems are extensions to $\mathbb{R}^{d}$-actions of the notion of subshift (see $[\mathrm{Ro}$ ). Classical examples are those generated by self-similar tilings, as the Penrose one, which have been extensively studied since the 90's. For details and references see for example Ro, So1. Systems arising from self-similar tilings are known to be linearly repetitive (see [So2, Lemma 2.3]); this means there exists a positive constant $L$ such that every pattern of diameter $D$ appears in every ball of radius $L D$ in any tiling of the system. This concept was first defined in $[\mathrm{LP}$. Linearly repetitive tiling and Delone systems can be seen as a generalization of the notion of linearly recurrent subshift to $\mathbb{R}^{d}$-actions, introduced in [DHS].

We study the factor maps between Delone systems. The main result is the following: linearly repetitive Delone systems have finitely many Delone system factors up to conjugacy. As noticed in $\underline{\mathrm{So} 3}$, tiling systems are topologically conjugate to Delone systems. This conjugacy also preserves linear repetitivity. Consequently, the results that we present can be easily extended to linearly repetitive tiling systems.

The main result of this paper was obtained in the context of subshifts in Du1. A key tool used in Du1 is the existence of sliding-block-codes for factor maps between subshifts (Curtis-Hedlund-Lyndon Theorem). Unlike subshifts, factor maps between two tiling systems are not always sliding-block-codes (see [Pe and $[\mathrm{RS}]$ ). The lack of this property appears to be the main difficulty of this work. To surmount this obstacle, we carefully dissect the continuity of factor maps by means of Voronoï cells and return vectors.

This paper is organized as follows: In Section 2 we recall basic concepts and results about Delone systems. In Section 3 we show the factor maps from linearly

Received by the editors December 8, 2008, and, in revised form, July 29, 2009.

2010 Mathematics Subject Classification. Primary 37B50.

Key words and phrases. Delone sets, tiling systems, factor maps, linearly repetitive, Voronoï cell.

(C)2009 American Mathematical Society Reverts to public domain 28 years from publication 
repetitive Delone systems to Delone systems are finite-to-one. Finally, Section 4 is devoted to the proof of the main theorem.

\section{Definitions And BaCKGRound}

In this section we give the basic definitions and properties concerning Delone sets. For more details we refer to $\underline{\mathrm{LP}}$ and $\underline{\mathrm{Ro}}$. Let $r$ and $R$ be two positive real numbers. An $(r, R)$-Delone set $X$ is a discrete subset of $\mathbb{R}^{d}$ satisfying the following two properties:

(1) Uniform discreteness: each open ball of radius $r>0$ in $\mathbb{R}^{d}$ contains at most one point of $X$.

(2) Relative density: each closed ball of radius $R>0$ in $\mathbb{R}^{d}$ contains at least one point of $X$.

An $(r, R)$-Delone set $X$, in short a Delone set, has finite local complexity if $X-X$ is locally finite; i.e. the intersection of $X-X$ with any bounded set is finite.

The translation by a vector $v \in \mathbb{R}^{d}$ of a Delone set $X$ is the Delone set $X-v$ obtained after translating every point of $X$ by $-v$. Observe that $X-v$ has finite local complexity if and only if $X$ has finite local complexity. A Delone set is said to be nonperiodic if $X-v=X$ implies $v=0$.

Let $R>0$ and let $X$ be a Delone set. We say that $P \subseteq X$ is the $R$-patch of $X$ centered at the point $y \in \mathbb{R}^{d}$ if

$$
P=X \cap B_{R}(y),
$$

where $B_{R}(y)$ denotes the open ball of a radius $R$ centered at $y$. If there is no confusion, we refer to an $R$-patch of $X$ merely as a patch. A sub-patch of the patch $P$ is a patch of $X$ included in $P$. A patch $Q$ is a translation of the patch $P$ if there exists $v \in \mathbb{R}^{d}$ such that $P-v=Q$. The vector $v \in \mathbb{R}^{d}$ is a return vector of the patch $P$ in $X$ if $P-v$ is a patch of $X$. An occurrence of the patch $P$ of $X$ centered at $y \in \mathbb{R}^{d}$ is a point $w \in \mathbb{R}^{d}$ such that $y-w$ is a return vector of $P$. Observe that the patch $P-(y-w)$ is the translation of $P$ centered at $w$.

The $R$-atlas $\mathcal{A}_{X}(R)$ of $X$ is the collection of all the $R$-patches centered at a point of $X$ translated to the origin. More precisely:

$$
\mathcal{A}_{X}(R)=\left\{X \cap B_{R}(x)-x ; x \in X\right\} .
$$

The atlas $\mathcal{A}_{X}$ of $X$ is the union of all the $R$-atlases, for $R>0$. Notice that $X$ has finite local complexity if and only if $\mathcal{A}_{X}(R)$ has finite local complexity for every $R>0$.

The Delone set $X$ is repetitive if for each $R>0$ there is a finite number $M>0$, such that for every closed ball $B$ of radius $M$ the set $B \cap X$ contains a translated patch of every $R$-patch of $X$. Observe that any repetitive Delone set has necessarily finite local complexity.

The Voronoï cell of a point $x \in X$ is the compact subset

$$
V_{x}=\left\{y \in \mathbb{R}^{d} ;\|x-y\| \leq\left\|x^{\prime}-y\right\| \text { for any } x^{\prime} \in X\right\} .
$$

Notice that if $X$ is a Delone set that has finite local complexity, then each Voronoï cell of $X$ is a polyhedra, and there is a finite number of Voronoï cells of $X$ up to translations. 
2.1. Delone systems. We denote by $\mathcal{D}$ the collection of the Delone sets of $\mathbb{R}^{d}$. The group $\mathbb{R}^{d}$ acts on $\mathcal{D}$ by translations:

$$
(v, X) \mapsto X-v \text { for } v \in \mathbb{R}^{d} \text { and } X \in \mathcal{D} .
$$

Furthermore, this action is continuous with the topology induced by the following distance: take $X, X^{\prime}$ in $\mathcal{D}$, and define $A$ as the set of $\varepsilon \in\left(0, \frac{1}{\sqrt{2}}\right)$ such that there exist $v$ and $v^{\prime}$ in $B_{\varepsilon}(0)$ with

$$
(X-v) \cap B_{1 / \varepsilon}(0)=\left(X^{\prime}-v^{\prime}\right) \cap B_{1 / \varepsilon}(0) ;
$$

we set

$$
d\left(X, X^{\prime}\right)=\left\{\begin{array}{cc}
\inf A & \text { if } A \neq \emptyset \\
\frac{1}{\sqrt{2}} & \text { if } A=\emptyset .
\end{array}\right.
$$

Roughly speaking, two Delone sets are close if they have the same pattern in a large neighborhood of the origin, up to a small translation.

A Delone system is a pair $\left(\Omega, \mathbb{R}^{d}\right)$ such that $\Omega$ is a translation invariant closed subset of $\mathcal{D}$. The orbit closure of a Delone set $X$ in $\mathcal{D}$ is the set $\Omega_{X}=\overline{\left\{X+v: v \in \mathbb{R}^{d}\right\}}$. This is invariant by the $\mathbb{R}^{d}$-action, and it is compact if and only if $X$ has finite local complexity (see $\left[\mathrm{Ro}\right.$ and $[\mathrm{Ru}]$ ). Every $X^{\prime} \in \Omega_{X}$ is an $(r, R)$-Delone set if $X$ is an $(r, R)$-Delone set, and for any real $R>0$, we have $\mathcal{A}_{X^{\prime}}(R) \subset \mathcal{A}_{X}(R)$. If all the orbits are dense in $\Omega_{X}$, the Delone system $\left(\Omega_{X}, \mathbb{R}^{d}\right)$ is said to be minimal. It is shown in $[\mathrm{Ro}$, that the Delone set $X$ is repetitive if and only if the system $\left(\Omega_{X}, \mathbb{R}^{d}\right)$ is minimal. In that case, for any $X^{\prime} \in \Omega_{X}$ and any $R>0$ the $R$-atlases $\mathcal{A}_{X^{\prime}}(R), \mathcal{A}_{X}(R)$ are the same. If in addition $X$ is nonperiodic, then every Delone set in $\Omega_{X}$ is nonperiodic.

A factor map between two Delone systems $\left(\Omega_{1}, \mathbb{R}^{d}\right)$ and $\left(\Omega_{2}, \mathbb{R}^{d}\right)$ is a continuous surjective map $\pi: \Omega_{1} \rightarrow \Omega_{2}$ such that $\pi(X-v)=\pi(X)-v$, for every $X \in \Omega_{1}$ and $v \in \mathbb{R}^{d}$.

In symbolic dynamics it is well-known that topological factor maps between subshifts are always given by sliding-block-codes. There are examples which show that this result cannot be extended to Delone systems ([Pe, $[\mathrm{RS}]$ ). The following lemma shows that factor maps between Delone systems are not far from being sliding-block-codes. A similar result can be found in HRS.

Lemma 1. Let $X_{1}$ and $X_{2}$ be two Delone sets. Suppose $X_{1}$ has finite local complexity and $\pi: \Omega_{X_{1}} \rightarrow \Omega_{X_{2}}$ is a factor map. Then, there exists a constant $s_{0}>0$ such that for every $\varepsilon>0$, there exists $R_{\varepsilon}>0$ satisfying the following: For any $R \geq R_{\varepsilon}$, if $X$ and $X^{\prime}$ in $\Omega_{X_{1}}$ verify

$$
X \cap B_{R+s_{0}}(0)=X^{\prime} \cap B_{R+s_{0}}(0) ;
$$

then

$$
(\pi(X)-v) \cap B_{R}(0)=\pi\left(X^{\prime}\right) \cap B_{R}(0)
$$

for some $v \in B_{\varepsilon}(0)$.

Proof. The Delone set $X_{2}$ also has finite local complexity because $\Omega_{X_{2}}$ is compact. Let $r_{0}$ and $R_{0}$ be positive constants such that $X_{2}$ is an $\left(r_{0}, R_{0}\right)$-Delone set. Since all the elements of $\Omega_{X_{2}}$ are $\left(r_{0}, R_{0}\right)$-Delone sets, if two different points $y_{1}, y_{2}$ of $\mathbb{R}^{d}$ satisfy $\left(X-y_{1}\right) \cap B_{R}(a)=\left(X-y_{2}\right) \cap B_{R}(a)$ for some $X \in \Omega_{X_{2}}, a \in \mathbb{R}^{d}$ and $R>R_{0}$, then $\left\|y_{1}-y_{2}\right\| \geq \frac{r_{0}}{2}$ (for the details see [So1] $)$. 
Let $0<\delta_{0}<\min \left\{\frac{r_{0}}{4}, \frac{1}{R_{0}}\right\}$. Since $\pi$ is uniformly continuous, there exists $s_{0}>1$ such that if $X$ and $X^{\prime}$ in $\Omega_{X_{1}}$ verify $X \cap B_{s_{0}}(0)=X^{\prime} \cap B_{s_{0}}(0)$, then

$$
(\pi(X)-v) \cap B_{\frac{1}{\delta_{0}}}(0)=\pi\left(X^{\prime}\right) \cap B_{\frac{1}{\delta_{0}}}(0),
$$

for some $v \in B_{\delta_{0}}(0)$. Let $0<\varepsilon<\delta_{0}$. By uniform continuity of $\pi$, there exists $0<\delta<\frac{1}{s_{0}}$ such that if $X$ and $X^{\prime}$ in $\Omega_{X_{1}}$ verify $X \cap B_{\frac{1}{\delta}}(0)=X^{\prime} \cap B_{\frac{1}{\delta}}(0)$, then

$$
(\pi(X)-v) \cap B_{\frac{1}{\varepsilon}}(0)=\pi\left(X^{\prime}\right) \cap B_{\frac{1}{\varepsilon}}(0),
$$

for some $v \in B_{\varepsilon}(0)$. Now fix $R \geq R_{\varepsilon}=\frac{1}{\delta}-s_{0}$, and let $X$ and $X^{\prime}$ be two Delone sets in $\Omega_{X_{1}}$ satisfying

$$
X \cap B_{R+s_{0}}(0)=X^{\prime} \cap B_{R+s_{0}}(0) .
$$

Observe that $X$ and $X^{\prime}$ satisfy (2.1), and $(X-a) \cap B_{s_{0}}(0)=\left(X^{\prime}-a\right) \cap B_{s_{0}}(0)$, for every $a$ in $B_{R}(0)$. The choice of $s_{0}$ ensures that

$$
(\pi(X)-a-t(a)) \cap B_{\frac{1}{\delta_{0}}}(0)=\left(\pi\left(X^{\prime}\right)-a\right) \cap B_{\frac{1}{\delta_{0}}}(0),
$$

for some $t(a) \in B_{\delta_{0}}(0)$. Let us prove the map $a \rightarrow t(a)$ is locally constant. For $a \in B_{R}(0)$, let $0<s_{a}<\frac{1}{\delta_{0}}-R_{0}$ be such that $B_{s_{a}}(a) \subseteq B_{R}(0)$. Every $a^{\prime} \in B_{s_{a}}(0)$ verifies $B_{\frac{1}{\delta_{0}}-\left\|a^{\prime}\right\|}\left(-a^{\prime}\right) \subset B_{\frac{1}{\delta_{0}}}(0)$. Let $a^{\prime} \in B_{s_{a}}(0)$. This inclusion and (2.3) imply

$$
\left(\pi(X)-a-a^{\prime}-t(a)\right) \cap B_{\frac{1}{\delta_{0}}-\left\|a^{\prime}\right\|}\left(-a^{\prime}\right)=\left(\pi\left(X^{\prime}\right)-a-a^{\prime}\right) \cap B_{\frac{1}{\delta_{0}}-\left\|a^{\prime}\right\|}\left(-a^{\prime}\right) .
$$

On the other hand, from the definition of the map $a \rightarrow t(a)$ we deduce

$$
\left(\pi(X)-a-a^{\prime}-t\left(a+a^{\prime}\right)\right) \cap B_{\frac{1}{\delta_{0}}}(0)=\left(\pi\left(X^{\prime}\right)-a-a^{\prime}\right) \cap B_{\frac{1}{\delta_{0}}}(0),
$$

which implies

$$
\left(\pi(X)-a-a^{\prime}-t\left(a+a^{\prime}\right)\right) \cap B_{\frac{1}{\delta_{0}}-\left\|a^{\prime}\right\|}\left(-a^{\prime}\right)=\left(\pi\left(X^{\prime}\right)-a-a^{\prime}\right) \cap B_{\frac{1}{\delta_{0}}-\left\|a^{\prime}\right\|}\left(-a^{\prime}\right) .
$$

Since $\left\|t(a)-t\left(a+a^{\prime}\right)\right\| \leq \frac{r_{0}}{2}$, from equations (2.4), (2.5) and the remark at the beginning of the proof we conclude $t(a)=t\left(a+a^{\prime}\right)$ for every $a^{\prime} \in B_{s}(0)$. Therefore the map $a \mapsto t(a)$ is constant on $B_{s_{a}}(a)$.

Furthermore, due to $\delta_{0}>\varepsilon$ and (2.2), equation (2.1) implies there exists $v \in$ $B_{\varepsilon}(0)$ such that

$$
(\pi(X)-v) \cap B_{\frac{1}{\delta_{0}}}(0)=\pi\left(X^{\prime}\right) \cap B_{\frac{1}{\delta_{0}}}(0) .
$$

For $a=0$, from (2.3) and (2.6) we have that $t(0)=v$ or $\|v-t(0)\| \geq \frac{r_{0}}{2}$. Since $\|t(0)-v\| \leq \delta_{0}+\varepsilon<2 \delta_{0}<\frac{r_{0}}{2}$, we conclude $t(0)=v$ and then $t(a)=v$ for every $a \in B_{R}(0)$. This property together with (2.3) and (2.6) imply that

$$
(\pi(X)-v) \cap B_{R}(0)=\pi\left(X^{\prime}\right) \cap B_{R}(0) .
$$

This concludes the proof.

\section{Preimages of FaCtor MAPS}

In the rest of this paper we suppose that all the Delone sets have finite local complexity.

A Delone set $X$ is linearly repetitive if there exists a constant $L>0$ such that for every patch $P$ in $X$, any ball of radius $L \operatorname{diam}(P)$ intersected with $X$ contains a translated patch of $P$. In this instance we say that $X$ is linearly repetitive with constant $L$. Notice the constant $L$ must be greater than or equal to 1 , and if $X$ is linearly repetitive with constant $L$, then it is linearly repetitive with constant $L^{\prime}$, for 
every $L^{\prime}>L$. Every Delone set in the orbit closure of a linearly repetitive Delone set is linearly repetitive with the same constant. When $X$ is linearly repetitive, we call $\left(\Omega_{X}, \mathbb{R}^{d}\right)$ a linearly repetitive Delone system.

The following lemma shows the factors of linearly repetitive systems are also linearly repetitive with a uniform control on the constants. This was already proven for subshifts in Du1.

Lemma 2. Let $X$ be a linearly repetitive Delone set with constant $L$. If $X^{\prime}$ is a Delone set such that $\left(\Omega_{X^{\prime}}, \mathbb{R}^{d}\right)$ is a topological factor of $\left(\Omega_{X}, \mathbb{R}^{d}\right)$, then there exists a constant $\tau_{X^{\prime}}>0$ such that if $P$ is a patch of $X^{\prime}$ with $\operatorname{diam}(P) \geq \tau_{X^{\prime}}$, then for any $y \in \mathbb{R}^{d}$, the set $X^{\prime} \cap B_{5 L \operatorname{diam}(P)}(y)$ contains a translated patch of $P$.

Proof. Let $\pi: \Omega_{X} \rightarrow \Omega_{X^{\prime}}$ be a topological factor, where $X$ is an $\left(r_{X}, R_{X}\right)$-linearly repetitive Delone set with constant $L$ and $X^{\prime}$ is an $\left(r_{X^{\prime}}, R_{X^{\prime}}\right)$-Delone set. We can assume that $\pi(X)=X^{\prime}$. Let $s_{0}>0$ be the constant of Lemma 1. Fix $0<\varepsilon<L s_{0}$ and consider $R_{\varepsilon}>0$ as in Lemma 1 We set

$$
\tau_{X^{\prime}}=\max \left\{s_{0}, R_{\varepsilon}, R_{X}, R_{X^{\prime}}\right\} .
$$

Let $P$ be a patch in $X^{\prime}$ with $\operatorname{diam}(P)=D \geq \tau_{X^{\prime}}$, and let $v \in P \subset X^{\prime}$. Let $Q=(X-v) \cap B_{D+s_{0}}(0)$. Since $\operatorname{diam}(Q) \leq 2\left(D+s_{0}\right)$, for every $y \in \mathbb{R}^{d}$ there exists $w \in B_{2 L\left(D+s_{0}\right)}(y)$ such that $(X-w) \cap B_{D+s_{0}}(0)=Q$. Then, from Lemma 1 there exists $t \in B_{\varepsilon}(0)$ such that

$$
\left(X^{\prime}-v\right) \cap B_{D}(0)=\left(X^{\prime}-w-t\right) \cap B_{D}(0) .
$$

Since $\left(X^{\prime}-v\right) \cap B_{D}(0)$ contains a translation of $P$, this shows that every ball of radius $2 L\left(D+s_{0}\right)+\varepsilon \leq 5 L D$ in $X^{\prime}$ contains a translation of $P$ as a sub-patch.

The next lemma follows the same lines as Lemma 2.4 in $\underline{\text { So2 }}$. We show that the set of occurrences of an $R$-patch of a linearly repetitive Delone set and its factors are uniformly discrete with a constant depending linearly on $R$.

Lemma 3. Let $X$ be a nonperiodic linearly repetitive Delone set with constant $L$, and let $X^{\prime}$ be a nonperiodic Delone set such that $\left(\Omega_{X^{\prime}}, \mathbb{R}^{d}\right)$ is a topological factor of $\left(\Omega_{X}, \mathbb{R}^{d}\right)$. There exists a constant $M_{X^{\prime}}>0$ such that for every $R \geq M_{X^{\prime}}$ and for every $R$-patch $P$ of $X^{\prime}$, if $x \in \mathbb{R}^{d} \backslash\{0\}$ is a return vector of $P$, then $\|x\| \geq R /(11 L)$.

Proof. Let $R^{\prime}>0$ be a real number such that any patch of the kind $X^{\prime} \cap B_{R^{\prime}}(y)$, with $y \in \mathbb{R}^{d}$, has diameter greater than $\tau_{X^{\prime}}$, where $\tau_{X^{\prime}}$ is the constant given by Lemma 2 Let $M_{X^{\prime}}=110 L R^{\prime}+R^{\prime}$ and let $P$ be the $R$-patch $X^{\prime} \cap B_{R}(v)$ with $R>M_{X^{\prime}}$ and $v \in \mathbb{R}^{d}$. Suppose there exists $x \in \mathbb{R}^{d}$, with $0<\|x\|<R /(11 L)$, such that $P+x$ is a patch of $X^{\prime}$. For any $y \in \mathbb{R}^{d}$, consider the patches

$$
Q_{y}=X^{\prime} \cap B_{R^{\prime}}(y) \text { and } S_{y}=X^{\prime} \cap B_{R^{\prime}+\|x\|}(y) .
$$

Since

$$
\tau_{X^{\prime}} \leq \operatorname{diam}\left(S_{y}\right) \leq 2\left(R^{\prime}+\|x\|\right),
$$

from Lemma 2, every ball of radius $10 L\left(R^{\prime}+\|x\|\right)$ intersected with $X^{\prime}$ contains a translation of $S_{y}$. By the very hypothesis, we have

$$
10 L\left(R^{\prime}+\|x\|\right)<10 L R^{\prime}+\frac{10 R}{11} \leq \frac{R}{11}+\frac{10 R}{11}=R .
$$


This implies there exists $w \in \mathbb{R}^{d}$ such that $S_{y}+w$ is a sub-patch of $X^{\prime} \cap B_{R}(v)=$ $P$. Because $P+x$ is also a patch of $X^{\prime}$, we have $Q_{y}+w+x$ is also a patch of $X^{\prime}$ and a sub-patch of $S_{y}+w$. Hence $Q_{y}+w+x=Q_{y+x}+w$ and

$$
Q_{y}+x=Q_{y+x}
$$

Since $y$ is arbitrary, we conclude that $X^{\prime}+x=X^{\prime}$, which contradicts the nonperiodicity of $X^{\prime}$ if $x \neq 0$.

We recall the following definition: A factor map $\pi:\left(\Omega, \mathbb{R}^{d}\right) \rightarrow\left(\Omega^{\prime}, \mathbb{R}^{d}\right)$ is said to be finite-to-one (with constant $D$ ) if for all $y \in Y$ we have $\left|\pi^{-1}(\{y\})\right| \leq D$.

The next result is a technical lemma we use in Proposition 5 to show that factor maps between linearly repetitive Delone systems are finite-to-one.

Lemma 4. Let $\pi:\left(\Omega_{X}, \mathbb{R}^{d}\right) \rightarrow\left(\Omega_{X^{\prime}}, \mathbb{R}^{d}\right)$ be a factor map, where $X$ is a linearly repetitive Delone set with constant $L$ and $X^{\prime}$ is a nonperiodic Delone set. We denote by $s_{0}$ the constant given by Lemma 1 .

For every $0<\varepsilon<\frac{s_{0}}{2}$, there exists a constant $R_{\pi}$ such that for any $R>R_{\pi}$ there are at most $n \leq\left(55 L^{2}\right)^{d}$ patches $P_{1}, \ldots, P_{n}$ satisfying for every $1 \leq i \leq n$ the following conditions:

i) $P_{i}=\left(X-w_{i}\right) \cap B_{R+s_{0}}(0)$, for some $w_{i} \in \mathbb{R}^{d}$.

ii) If $X^{\prime \prime}$ belongs to $\Omega_{X}$ and $X^{\prime \prime} \cap B_{R+s_{0}}(0)=P_{i}$, then there exists $v \in B_{\epsilon}(0)$ such that

$$
\left(\pi\left(X^{\prime \prime}\right)-v\right) \cap B_{R}(0)=\pi(X) \cap B_{R}(0) .
$$

iii) The patch $\left(X-w_{i}\right) \cap B_{R+s_{0}-2 \epsilon}(0)$ is not a sub-patch of $P_{j}$, for every $1 \leq$ $j \leq n, j \neq i$.

Proof. Let $0<\varepsilon<\frac{s_{0}}{2}, R_{\pi}=\max \left\{s_{0}, M_{X^{\prime}}, R_{\epsilon}\right\}$ and $R>R_{\pi}$, where $M_{X^{\prime}}$ is the constant given by Lemma 3 and $R_{\epsilon}$ by Lemma 1 . Let $P_{1}, \ldots, P_{n}$ be $n$ patches of $X$ satisfying the conditions $i$ ), ii), iii).

Let $1 \leq i \leq n$. We have

$$
\operatorname{diam}\left(P_{i}\right) \leq 2\left(R+s_{0}\right) \leq 4 R .
$$

Linear repetitivity implies there exists $v_{i} \in B_{4 L R}(0)$ such that

$$
\left(X-v_{i}\right) \cap B_{R+s_{0}}(0)=P_{i} .
$$

Then by $i i)$, there is $u_{i} \in B_{\epsilon}(0)$ satisfying

$$
Q=\left(\pi\left(X-v_{i}\right)+u_{i}\right) \cap B_{R}(0)=\left(\pi(X)-v_{i}+u_{i}\right) \cap B_{R}(0),
$$

where $Q=\pi(X) \cap B_{R}(0)$ (observe that $Q$ does not depend on $i$ ). This means the set $Q+v_{i}-u_{i}$ is a patch of $\pi(X)$. As $\left\{v_{i}-u_{i}, 1 \leq i \leq n\right\}$ is included in $B_{4 L R+\epsilon}(0)$ and $R>M_{X^{\prime}}$, Lemma 3 implies the number of elements in $\left\{v_{i}-u_{i}, 1 \leq i \leq n\right\}$ is bounded by

$$
\frac{\operatorname{vol}\left(B_{4 L R+\epsilon}(0)\right)}{\operatorname{vol}\left(B_{\frac{R}{11 L}}(0)\right)} \leq\left(55 L^{2}\right)^{d} .
$$

If $n$ is greater than $\left(55 L^{2}\right)^{d}$, then there exist $i \neq j$ such that $v_{i}-u_{i}=v_{j}-u_{j}$ and $\left\|v_{i}-v_{j}\right\|<2 \epsilon$. This implies that the patch $\left(X-v_{i}\right) \cap B_{R+s_{0}-2 \epsilon}(0)$ is included in the patch $\left(X-v_{j}\right) \cap B_{R+s_{0}}(0)=P_{j}$, which contradicts condition $\left.i i i\right)$.

The next result was proven in Du1 for subshifts. We use it with Proposition 6 to conclude the proof of the main theorem. 
Proposition 5. Let $X$ be a linearly repetitive Delone set with constant L. If $\pi:\left(\Omega_{X}, \mathbb{R}^{d}\right) \rightarrow\left(\Omega_{X^{\prime}}, \mathbb{R}^{d}\right)$ is a factor map such that $X^{\prime}$ is a nonperiodic Delone set, then $\pi$ is finite-to-one with constant $\left(55 L^{2}\right)^{d}$.

Proof. Let $X_{0}^{\prime} \in \Omega_{X^{\prime}}$. Suppose there exist $n>\left(55 L^{2}\right)^{d}$ elements $X_{1}, \ldots, X_{n}$ of $\Omega_{X}$ such that $\pi\left(X_{i}\right)=X_{0}^{\prime}$, for each $1 \leq i \leq n$. Since they are all different, there exists $R_{0}>0$ such that for any $R \geq R_{0}$, the patches $X_{i} \cap B_{R}(0)$ are pairwise distinct.

Let $0<\varepsilon<\frac{s_{0}}{2}$ and let $R_{\pi}$ be the constant given by Lemma 4. Lemma 1 ensures that for any $Y \in \Omega_{X}$ satisfying $Y \cap B_{R}(0)=X_{i} \cap B_{R}(0)$, with $1 \leq i \leq n$ and $R>\max \left\{R_{0}, R_{\epsilon}+s_{0}, R_{\pi}+s_{0}\right\}$, there exists $v \in B_{\epsilon}(0)$ such that $(\pi(Y)-v) \cap$ $B_{R-s_{0}}(0)=X_{0}^{\prime} \cap B_{R-s_{0}}(0)$. This means the patches $X_{1} \cap B_{R}(0), \cdots, X_{n} \cap B_{R}(0)$ satisfy conditions $i$ ) and $i$ ) of Lemma 4 Then we deduce that there exist different $i(R)$ and $j(R)$ in $\{1, \ldots, n\}$ such that the patch $X_{i(R)} \cap B_{R-2 \epsilon}(0)$ is a sub-patch of $X_{j(R)} \cap B_{R}(0)$. In other words, there exists $v_{R} \in B_{2 \epsilon}(0)$ such that $X_{i(R)} \cap$ $B_{R-2 \epsilon}(0)=\left(X_{j(R)}+v_{R}\right) \cap B_{R-2 \epsilon}(0)$.

By the pigeonhole principle, there exist different $i_{0}$ and $j_{0}$ in $\{1, \ldots, n\}$ and an increasing sequence $\left(R_{p}\right)_{p \geq 0}$, tending to $\infty$ with $p$, such that $i\left(R_{p}\right)=i_{0}$ and $j\left(R_{p}\right)=j_{0}$, for every $p \geq 0$. By compactness, we can also assume that $\left(v_{R_{p}}\right)_{p \geq 0}$ converges to a vector $v$. Thus, for every $p \geq 0$ we get

$$
X_{i_{0}} \cap B_{R_{p}-2 \epsilon}(0)=\left(X_{j_{0}}+v_{R_{p}}\right) \cap B_{R_{p}-2 \epsilon}(0),
$$

which implies that $X_{i_{0}}=X_{j_{0}}+v$ and $X_{0}^{\prime}=\pi\left(X_{i_{0}}\right)=\pi\left(X_{j_{0}}+v\right)=X_{0}^{\prime}+v$. Since $X_{i_{0}} \neq X_{j_{0}}$, the vector $v$ is different from zero, but this contradicts the nonperiodicity of $X_{0}^{\prime}$.

The following proposition is a straightforward generalization of Lemma 21 in Du1.

Proposition 6. Let $\left(\Omega, \mathbb{R}^{d}\right)$ be a minimal Delone system and let $\phi_{1}:\left(\Omega, \mathbb{R}^{d}\right) \rightarrow$ $\left(\Omega_{1}, \mathbb{R}^{d}\right), \phi_{2}:\left(\Omega, \mathbb{R}^{d}\right) \rightarrow\left(\Omega_{2}, \mathbb{R}^{d}\right)$ be two factor maps. Suppose that $\left(\Omega_{2}, \mathbb{R}^{d}\right)$ is nonperiodic and $\phi_{1}$ is finite-to-one. If there exist $X, Y \in \Omega$ and $v \in \mathbb{R}^{d}$ such that $\phi_{1}(X)=\phi_{1}(Y)$ and $\phi_{2}(X)=\phi_{2}(Y-v)$, then $v=0$.

Proof. There exists a sequence $\left(v_{i}\right)_{i \in \mathbb{N}} \subset \mathbb{R}^{d}$ such that $\lim _{i \rightarrow+\infty} X-v_{i}=Y$. By compactness, we can suppose that the sequence $\left(Y-v_{i}\right)_{i \in \mathbb{N}}$ converges to a point $Y_{2} \in \Omega$. By continuity, we have $\phi_{1}(Y)=\phi_{1}\left(Y_{2}\right)$ and $\phi_{2}(Y)=\phi_{2}\left(Y_{2}\right)-v$. By compactness, we can suppose that the sequence of points $\left(Y_{2}-v_{i}\right)_{i \in \mathbb{N}} \subset \Omega$ converges to a point $Y_{3}$. So we have $\phi_{1}\left(Y_{2}\right)=\phi_{1}\left(Y_{3}\right)$ and $\phi_{2}\left(Y_{2}\right)=\phi_{2}\left(Y_{3}\right)-v$. Hence we construct by induction a sequence $\left(Y_{n}\right)_{n \in \mathbb{N}} \subset \Omega$ such that $\phi_{1}\left(Y_{n}\right)=\phi_{1}\left(Y_{n+1}\right)$ and $\phi_{2}\left(Y_{n}\right)=\phi_{2}\left(Y_{n+1}\right)-v$ for all $n \geq 1$. Since the map $\phi_{1}$ is finite-to-one, there exist $i<j$ such that $Y_{i}=Y_{j}$. Then, we have

$$
\begin{aligned}
\phi_{2}\left(Y_{i}\right)=\phi_{2}\left(Y_{i+1}\right)-v=\phi_{2}\left(Y_{i+2}\right)-2 v=\ldots & =\phi_{2}\left(Y_{j}\right)-(j-i) v \\
& =\phi_{2}\left(Y_{i}\right)-(j-i) v .
\end{aligned}
$$

Since $\left(\Omega_{2}, \mathbb{R}^{d}\right)$ is nonperiodic, we conclude $v=0$.

Remark. Following the lines of the proof of Proposition [6, this result can be generalized to $\mathbb{Z}^{d}$ or $\mathbb{R}^{d}$ actions; more precisely: Let $G$ be $\mathbb{R}^{d}$ or $\mathbb{Z}^{d}$. Let $(X, G)$ be a minimal dynamical system and let $\phi_{1}:(X, G) \rightarrow\left(X_{1}, G\right), \phi_{2}:(X, G) \rightarrow\left(X_{2}, G\right)$ be two factor maps. Suppose that $\left(X_{2}, G\right)$ is free and $\phi_{1}$ is finite-to-one. If there exist $x, y \in X$ and $g \in G$ such that $\phi_{1}(x)=\phi_{1}(y)$ and $\phi_{2}(x)=\phi_{2}(g . y)$, then $g$ is the identity in $G$. 


\section{Number of FACTORS OF LINEARLY REPETITIVE Delone SYSTEMS}

Let $X$ be a Delone set having finite local complexity, and let $P=X \cap B_{R}(x)$ be a patch of $X$. We define

$$
X_{P}=\left\{v \in \mathbb{R}^{d}: P+v \text { is a patch of } X\right\} .
$$

Observe that 0 always belongs to $X_{P}$. It is straightforward to check that $X_{P}$ is a Delone set when $X$ is repetitive. Furthermore, $X_{P}$ is a Delone set having finite local complexity because of $X_{P}-X_{P} \subset X-X$. Then we define the Voronoï cell of $P$ associated to $v \in X_{P}$ as the Voronoï cell of $v+x \in X_{P}+x$. That is,

$$
V_{P, v}=\left\{y \in \mathbb{R}^{d}:\|y-(x+v)\| \leq\|y-(x+u)\|, \forall u \in X_{P}\right\} .
$$

Notice the Voronoï cell of $P$ associated to $v \in X_{P}$ is the Voronoï cell of $v \in X_{P}$ translated by the vector $x$.

Remark 7. It follows from the definition that an $(r, R)$-Delone set $X$ satisfies the following: for any $x \in X$, the diameter of the Voronoï cell $V_{x}$ is smaller than or equal to $2 R$ and $B_{\frac{r}{2}}(x)$ is contained in $V_{x}$. If $X$ is linearly repetitive with constant $L$, then Lemma 3 implies that for every sufficiently large $R$ and every patch $P=X \cap B_{R}(x)$ of $X$, the collection $X_{P}$ is an $\left(\frac{R}{11 L}, 2 L R\right)$-Delone set. Therefore, in this instance we have $\operatorname{diam}\left(V_{P, v}\right) \leq 4 L R$ and $B_{\frac{R}{11 L}}(x+v) \subseteq V_{P, v}$, for every $v \in X_{P}$.

In the next lemma, we restrict the number of ways we can extend a given patch $P$ to a bigger one. More precisely, we give an upper bound of the number (up to translation) of $R^{\prime}$-patches $X \cap B_{R^{\prime}}(x)$ such that $X \cap B_{R}(x)$ is a translation of $P$.

Lemma 8. Let $X$ be a linearly repetitive Delone set with constant $L$, and consider $0<R_{1}<R_{2}$, with $R_{1}$ sufficiently large. Then there are at most $n \leq\left(44 L^{2}\right)^{d}\left(\frac{R_{2}}{R_{1}}\right)^{d}$ patches $P_{1}, \cdots, P_{n}$ of $X$, up to translation, satisfying for every $1 \leq i \leq n$ the following two conditions:

i) There exists $v_{i} \in \mathbb{R}^{d}$ such that $P_{i}=X \cap B_{R_{2}}\left(v_{i}\right)$.

ii) $\left(X-v_{i}\right) \cap B_{R_{1}}(0)=\left(X-v_{j}\right) \cap B_{R_{1}}(0)$, for every $1 \leq j \leq n$.

Proof. Applying Lemma 3 to the identity factor map on $\left(\Omega_{X}, \mathbb{R}^{d}\right)$, we deduce that there exists $M_{X}>0$ such that for every $R \geq M_{X}$ and $x \in \mathbb{R}^{d}$, the distance between two different occurrences of $P=X \cap B_{R}(x)$ is greater than or equal to $R /(11 L)$.

Let $M_{X} \leq R_{1}<R_{2}$ and $n \in \mathbb{N}$. Suppose $P_{1}, \cdots, P_{n}$ are patches of $X$ satisfying conditions $i$ ) and $i i$ ) and such that for every $1 \leq i \leq n$,

iii) $P_{i}$ is not a translation of $P_{j}$, for every $j \in\{1, \cdots, n\} \backslash\{i\}$.

Condition $i$ ) and linear repetitivity of $X$ imply that for every $1 \leq i \leq n$ there exists $w_{i} \in \mathbb{R}^{d}$ such that $P_{i}+w_{i}$ is a sub-patch of $X \cap B_{2 L R_{2}}(0)$. From condition ii) it follows that for every $1 \leq i \leq n$, the point $v_{i}+w_{i}$ is an occurrence of the patch $X \cap B_{R_{1}}\left(v_{1}\right)$ in the ball $B_{2 L R_{2}}(0)$. Finally, by the choice of $R_{1}$, conditions ii), iii) and Lemma 3. for every $i$ and $j$ in $\{1, \cdots, n\}$ such that $i \neq j$, we get $\left\|v_{i}+w_{i}-\left(v_{j}+w_{j}\right)\right\| \geq \frac{R_{1}}{11 L}$, which implies

$$
n \leq \frac{\operatorname{vol}\left(B_{2 L R_{2}}(0)\right)}{\operatorname{vol}\left(B_{\frac{R_{1}}{22 L}}(0)\right)}=\left(44 L^{2}\right)^{d}\left(\frac{R_{2}}{R_{1}}\right)^{d}
$$

and achieves the proof. 
The following lemma is certainly well-known, but we did not find any reference. This shows that a Voronoi cell of a point $x$ in an $(r, R)$-Delone set $X$ is completely determined by the points in $X \cap B_{4 R}(x)$.

Lemma 9. Let $X$ be an $(r, R)$-Delone set. Then for every $x \in X$ one has

$$
V_{x}=\left\{y \in \mathbb{R}^{d}:\|x-y\| \leq\left\|x^{\prime}-y\right\|, \text { for every } x^{\prime} \in X \cap B_{4 R}(x)\right\} .
$$

Proof. Let $C_{x}=\left\{y \in \mathbb{R}^{d}:\|x-y\| \leq\left\|x^{\prime}-y\right\|\right.$, for every $\left.x^{\prime} \in X \cap B_{4 R}(x)\right\}$. By the definition of a Voronoï cell, the inclusion $V_{x} \subseteq C_{x}$ is direct.

Observe that the set $C_{x}$ is convex because it is obtained as the intersection of convex sets. Now, suppose there exists $y \in C_{x} \backslash V_{x}$. Then there exist $x^{\prime} \in X$, satisfying $V_{x} \cap V_{x^{\prime}} \neq \emptyset$, and $z \in\left([x, y] \cap V_{x^{\prime}}\right) \backslash V_{x}$, where $[x, y]$ is the segment with extreme points $x$ and $y$. Since $\left\|x-x^{\prime}\right\| \leq 4 R$ and $\left\|z-x^{\prime}\right\|<\|z-x\|$, the definition of $C_{x}$ implies $z \notin C_{x}$, which contradicts the convexity of $C_{x}$.

Lemma 10. Let $X$ be a nonperiodic linearly repetitive Delone set with constant L. There exists a positive constant $c(L)$ such that for every sufficiently large $R$ and every patch $P=X \cap B_{R}(x)$, the collection $\left\{X \cap V_{P, v}: v \in X_{P}\right\}$ contains at most $c(L)$ elements up to translation.

Proof. Let $R$ be a big enough positive number in order to apply Lemma 8 to $R_{1}=R$ and $R_{2}=8 L R$.

Let $x \in \mathbb{R}^{d}, P=X \cap B_{R}(x)$ and $v \in X_{P}$. Since $X_{P}+x$ is a Delone set with constant of uniform density equal to $2 L R$ (see Remark 7), Lemma 9 implies $V_{P, v}$ is completely determined by the patch $X \cap B_{8 R L}(v+x)$. Furthermore, the Voronoï cell $V_{P, v}$ is contained in the ball $B_{4 R L}(v+x)$ (see Remark 7). Then it follows there are at most as many Voronoï cells of $P$ and patches of the kind $X \cap V_{P, v}$, up to translation, as patches $Q$ satisfying the following two conditions: $i$ ) there exists $w \in \mathbb{R}^{d}$ such that $Q=X \cap B_{8 R L}(w)$ and $\left.i i\right) w$ is an occurrence of a translation of $P$. These two conditions and Lemma 8 imply there are at most

$$
c(L) \leq\left(44 L^{2}\right)^{d}\left(\frac{8 L R}{R}\right)^{d}=\left(352 L^{3}\right)^{d}
$$

patches of the kind $X \cap V_{P, v}$ up to translation.

We have already defined the notion of return vector of a patch. Now let us define the notion of return vector of a Voronoï cell of a patch. For a patch $P=$ $X \cap B_{R}(x)$ of $X$ and $v \in X_{P}$, we say that $w \in \mathbb{R}^{d}$ is a return vector of $V_{P, v} \cap X$ if $(X-w) \cap V_{P, v}=X \cap V_{P, v}$. We set

$$
P_{n, w, v} \text { the patch }(X-w-x-v) \cap B_{L^{n} R}(0) \text {. }
$$

Notice that $P_{n, w, v}+v+w+x$ is a patch of $X$. When there is no confusion about $n$ and $v$, we write $P_{w}$ instead of $P_{n, w, v}$.

Lemma 11. Let $n \in \mathbb{N}$ and let $X$ be a nonperiodic linearly repetitive Delone set with constant $L$. For every sufficiently large $R>0$ and every $R$-patch $P$, the collection $\left\{P_{w}: w\right.$ is a return vector of $\left.V_{P, v} \cap X\right\}$ has at most $c(n, L)$ elements for every $v \in X_{P}$.

Proof. Let $R_{1}=R$ and $R_{2}=L^{n} R$ be sufficiently large positive numbers in order to apply Lemma 8. Let $P=X \cap B_{R}(x)$ be a patch of $X$ and let $v \in X_{P}$. Since $X_{P}+x$ is a Delone set with constant of uniform discreteness equal to $\frac{R}{11 L}$, the Voronoï cell 
$V_{P, v}$ contains the ball $B_{\frac{R}{22}}(v+x)$. This implies that for every pair of return vectors $u$ and $w$ of $V_{P, v}$ it holds that $P_{w} \cap B_{\frac{R}{22}}(0)=P_{u} \cap B_{\frac{R}{22}}(0)$. Thus, from Lemma 8 it follows that there are at most

$$
c(n, L) \leq\left(44 L^{2}\right)^{d}\left(\frac{L^{n} R}{\frac{R}{22 L}}\right)^{d}=\left(968 L^{n+3}\right)^{d}
$$

patches of the kind $P_{w}$.

Let $n \in \mathbb{N}$. We call $M(n, L)$ the number of coverings of a set with $c(L) c(n, L)$ elements, where $c(L)$ and $c(n, L)$ are the constants of Lemma 10 and Lemma 11 respectively.

Theorem 12. Let $X$ be a linearly repetitive Delone set. There are finitely many Delone system factors of $\left(\Omega_{X}, \mathbb{R}^{d}\right)$ up to conjugacy. Moreover, the number of factors only depends on the linearly repetitive constant of $X$.

Proof. Let $X$ be a nonperiodic linearly repetitive Delone set with constant $L>1$. Let $n \in \mathbb{N}$ be such that

$$
L^{n}-1-12 L-176 L^{2}>1
$$

and let $R_{1}>1$ be a constant such that for every $R \geq R_{1}$, Lemma 10 and Lemma 11 are applicable to $R$-patches of $X$.

For every $1 \leq i \leq M(n, L)+1$, let $X_{i}$ be a nonperiodic Delone set such that there exists a topological factor map $\pi_{i}: \Omega_{X} \rightarrow \Omega_{X_{i}}$, and let $X_{0}=X$. Let $M_{X_{i}}$ be the constant of Lemma 3 associated to $X_{i}$.

Fix $0<\varepsilon<1$. For every $1 \leq i \leq M(n, L)+1$, consider $R_{\varepsilon}^{(i)}$ and $s_{0}^{(i)}$ as the constants of Lemma 1 associated to $\pi_{i}$. We define

$$
R_{\varepsilon}=\max _{i}\left\{R_{\varepsilon}^{(i)}\right\}, s_{0}=\max _{i}\left\{s_{0}^{(i)}\right\} \text { and } M=\max _{i}\left\{M_{X_{i}}\right\} .
$$

Observe in an open ball of radius $r / 22 L$ that there is at most one return vector of an $r$-patch of $X_{i}$, with $r \geq M$, for every $1 \leq i \leq M(n, L)+1$.

We take

$$
R>\max \left\{R_{\varepsilon}, s_{0}, M+\varepsilon, R_{1}, 45 L\right\} .
$$

Consider the patch $P=B_{R}(0) \cap X$, and let $v_{1}, \cdots, v_{N} \in X_{P}$ be such that for every $v \in X_{P}$, there exist $1 \leq i \leq N$ and $u \in \mathbb{R}^{d}$ satisfying $V_{P, v} \cap X=\left(V_{P, v_{i}} \cap X\right)+u$. Roughly speaking, every set of the kind $V_{P, v} \cap X$ is a translation of some set $V_{P, v_{i}} \cap X$. Since $R>R_{1}$, Lemma 10 ensures $N \leq c(L)$.

For every $1 \leq j \leq N$, let $w_{j, 1}, \cdots, w_{j, m_{j}}$ be return vectors of $V_{P, v_{j}} \cap X$, chosen so that for every return vector $w$ of $V_{P, v_{j}} \cap X$, there exists $1 \leq i \leq m_{j}$ such that $P_{w}$ is equal to $P_{w_{j, i}}$. Since $R>R_{1}$, Lemma 11 implies that $m_{j} \leq c(n, L)$, for every $1 \leq j \leq N$. Therefore, the collection

$$
\mathcal{F}=\left\{P_{w_{j, l}}: 1 \leq l \leq m_{j}, 1 \leq j \leq N\right\}
$$

contains at most $c(L) c(n, L)$ elements.

Let $R^{\prime}$ be the constant given by

$$
R^{\prime}=\left(L^{n}-1\right) R-\varepsilon-4 L R .
$$

The choice of $n$ ensures that $R^{\prime}>0$. 
For every $1 \leq i \leq M(n, L)+1$, we define the following relation on $\mathcal{F}: P_{w_{j, l}} \mathcal{R}_{i} P_{w_{k, m}}$ if and only if for every $X^{\prime}, X^{\prime \prime} \in \Omega_{X}$ such that $X^{\prime} \cap B_{L^{n} R}(0)=P_{w_{j, l}}$ and $X^{\prime \prime} \cap$ $B_{L^{n} R}(0)=P_{w_{k, m}}$, there exist $v \in B_{2 \varepsilon}(0)$ and $w \in B_{4 L R}(0)$ such that $\pi_{i}\left(X^{\prime}\right) \cap$ $B_{R^{\prime}}(0)=\left(\pi_{i}\left(X^{\prime \prime}\right)+v+w\right) \cap B_{R^{\prime}}(0)$.

Since $L^{n} R-s_{0} \geq\left(L^{n}-1\right) R \geq R>R_{\varepsilon}$, from Lemma 1 it follows that this relation is reflexive, and therefore nonempty. Since the cardinal of $\mathcal{F}$ is bounded by $c(L) c(n, L)$, there are at most $M(n, L)$ different relations of this kind. So, there exist $1 \leq i<j<M(n, L)+1$ such that $\mathcal{R}_{i}=\mathcal{R}_{j}$.

In the sequel, we will prove that $\left(\Omega_{X_{i}}, \mathbb{R}^{d}\right)$ and $\left(\Omega_{X_{j}}, \mathbb{R}^{d}\right)$ are conjugate. For that, it is sufficient to show that if $Y, Z \in \Omega_{X}$ are such that $\pi_{i}(Y)=\pi_{i}(Z)$, then $\pi_{j}(Y)=\pi_{j}(Z)$.

Let $Y$ and $Z$ be two Delone sets in $\Omega_{X}$ such that $\pi_{i}(Y)=\pi_{i}(Z)$. Without loss of generality, we can suppose that 0 is an occurrence of $P$ in $Y$ and in $Z-u_{0}$, where $u_{0}$ is some point in $B_{4 L R}(0)$. The patches of $Y$ and $Z$ are translations of the patches of $X$. This implies that there exist $1 \leq q_{0}, r_{0} \leq N$ such that

$$
Y \cap B_{L^{n} R}(0)=P_{w_{q_{0}, l_{0}}} \text { and }\left(Z-u_{0}\right) \cap B_{L^{n} R}(0)=P_{w_{r_{0}, k_{0}}},
$$

for some $1 \leq l_{0} \leq m_{q_{0}}$ and $1 \leq k_{0} \leq m_{r_{0}}$.

Claim 1: $P_{w_{q_{0}, l_{0}}} \mathcal{R}_{i} P_{w_{r_{0}, k_{0}}}$.

Proof of Claim 1. Let $X^{\prime}$ and $X^{\prime \prime}$ be two Delone sets in $\Omega_{X}$ such that $X^{\prime} \cap B_{L^{n}} R(0)$ $=P_{w_{q_{0}, l_{0}}}$ and $X^{\prime \prime} \cap B_{L^{n} R}(0)=P_{w_{r_{0}, l_{0}}}$. Since $R \geq s_{0}, R \geq R_{\varepsilon}$ and

$$
X^{\prime} \cap B_{L^{n} R}(0)=Y \cap B_{L^{n} R}(0), X^{\prime \prime} \cap B_{L^{n} R}(0)=\left(Z-u_{0}\right) \cap B_{L^{n} R}(0) .
$$

By the choice of $n$ and $R$, Lemma 1 implies that there exist $z_{1}$ and $z_{2}$ in $B_{\varepsilon}(0)$ such that

$$
\begin{aligned}
\left(\pi_{i}\left(X^{\prime}\right)+z_{1}\right) \cap B_{\left(L^{n}-1\right) R}(0) & =\pi_{i}(Y) \cap B_{\left(L^{n}-1\right) R}(0) \text { and } \\
\left(\pi_{i}\left(X^{\prime \prime}\right)+z_{2}\right) \cap B_{\left(L^{n}-1\right) R}(0) & =\pi_{i}\left(Z-u_{0}\right) \cap B_{\left(L^{n}-1\right) R}(0) .
\end{aligned}
$$

Then we get

$$
\begin{aligned}
& \left(\pi_{i}\left(X^{\prime \prime}\right)+z_{2}+u_{0}\right) \cap B_{\left(L^{n}-1\right) R-4 L R}(0) \\
= & \pi_{i}(Z) \cap B_{\left(L^{n}-1\right) R-4 L R}(0) \\
= & \pi_{i}(Y) \cap B_{\left(L^{n}-1\right) R-4 L R}(0) \\
= & \left(\pi_{i}\left(X^{\prime}\right)+z_{1}\right) \cap B_{\left(L^{n}-1\right) R-4 L R}(0) .
\end{aligned}
$$

Therefore

$$
\left(\pi_{i}\left(X^{\prime \prime}\right)+z_{2}+u_{0}-z_{1}\right) \cap B_{\left(L^{n}-1\right) R-4 L R-\varepsilon}(0)=\pi_{i}\left(X^{\prime}\right) \cap B_{\left(L^{n}-1\right) R-4 L R-\varepsilon}(0),
$$

which implies that $P_{w_{q_{0}, l_{0}}} \mathcal{R}_{i} P_{w_{r_{0}, k_{0}}}$.

Since $\mathcal{R}_{i}=\mathcal{R}_{j}$, from Claim 1 we get $P_{w_{q_{0}, l_{0}}} \mathcal{R}_{j} P_{w_{r_{0}, k_{0}}}$.

Let $s$ be any other occurrence of $P$ in $Y$. Repeating the same argument for $Y+s$ and $Z+s$, we deduce that there exist $u_{s} \in B_{4 L R}(0)$ and $1 \leq q_{s}, r_{s} \leq N$ such that

$$
(Y+s) \cap B_{L^{n} R}(0)=P_{w_{q_{s}, l_{s}}} \text { and }\left(Z-u_{s}\right) \cap B_{L^{n} R}(0)=P_{w_{r_{s}, k_{s}}},
$$

for some $1 \leq l_{s} \leq m_{q_{s}}$ and $1 \leq k_{s} \leq m_{r_{s}}$. From Claim 1 we get $P_{w_{q_{s}, l_{s}}} \mathcal{R}_{j} P_{w_{r_{s}, k_{s}}}$. This implies that there exist $t_{s} \in B_{2 \varepsilon}(0)$ and $w_{s} \in B_{4 L R}(0)$ such that

$$
\pi_{j}(Y+s) \cap B_{R^{\prime}}(0)=\left(\pi_{j}\left(Z+s-u_{s}\right)+t_{s}+w_{s}\right) \cap B_{R^{\prime}}(0) .
$$


Claim 2: The vector $w_{s}-u_{s}+t_{s}$ does not depend on $s$; i.e., there exists $y \in \mathbb{R}^{d}$ such that $w_{s}-u_{s}+t_{s}=y$ for every occurrence $s$ of $P$ in $Y$.

Proof of Claim 2. Let $s_{1}$ and $s_{2}$ be two occurrences of $P$ in $Y$ such that the Voronoï cells of $s_{1}$ and $s_{2}$, with respect to the set of occurrences of $P$ in $Y$, have common points in their borders. Since the diameter of these Voronoï cells is smaller than or equal to $4 R L$ (see Remark 7), we get $\left\|s_{1}-s_{2}\right\| \leq 8 L R$. Then

$$
\begin{aligned}
& \left(\pi_{j}(Z)+s_{1}+\left(s_{2}-s_{1}\right)-u_{s_{1}}+t_{s_{1}}+w_{s_{1}}\right) \cap B_{R^{\prime}-8 L R}(0) \\
= & \left(\pi_{j}(Y)+s_{1}+\left(s_{2}-s_{1}\right)\right) \cap B_{R^{\prime}-8 L R}(0) \\
= & \left(\pi_{j}(Z)+s_{2}-u_{s_{2}}+t_{s_{2}}+w_{s_{2}}\right) \cap B_{R^{\prime}-8 L R}(0) .
\end{aligned}
$$

This implies $\left(-u_{s_{1}}+t_{s_{1}}+w_{s_{1}}\right)-\left(-u_{s_{2}}+t_{s_{2}}+w_{s_{2}}\right)$ is a return vector of an $\left(R^{\prime}-8 L R\right)$ patch of $\pi_{j}(Z)+s_{2}$. Since

$$
R^{\prime}-8 L R=R\left(L^{n}-1-12 L\right)-\varepsilon \geq R-\varepsilon>M,
$$

Lemma 3 implies that the nonzero vectors of the $\left(R^{\prime}-8 L R\right)$-patches of $\pi_{j}(Z)+s_{2}$ have norm greater than or equal to $\left(R^{\prime}-8 L R\right) / 11 L$. Thus, due to

$$
\left\|-u_{s_{1}}+t_{s_{1}}+w_{s_{1}}-\left(-u_{s_{2}}+t_{s_{2}}+w_{s_{2}}\right)\right\| \leq 16 L R+4 \varepsilon
$$

and

$$
\begin{aligned}
11(16 L R+4 \varepsilon) & =176 L^{2} R+44 L \varepsilon \\
& <\left(L^{n}-1-12 L-1\right) R+44 L \varepsilon \\
& =R^{\prime}-8 L R+\varepsilon-R+44 L \varepsilon \\
& <R^{\prime}-8 L R+L-R+44 L<R^{\prime}-8 L R,
\end{aligned}
$$

we deduce $-u_{s_{1}}+t_{s_{1}}+w_{s_{1}}=-u_{s_{2}}+t_{s_{2}}+w_{s_{2}}$, which proves Claim 2 .

From Claim 2 we get that there exists $y \in \mathbb{R}^{d}$ such that for every occurrence $s$ of $P$ in $Y$,

$$
\pi_{j}(Y+s) \cap B_{R^{\prime}}(0)=\left(\pi_{j}(Z+s)+y\right) \cap B_{R^{\prime}}(0)
$$

and then

$$
\pi_{j}(Y) \cap B_{R^{\prime}}(s)=\left(\pi_{j}(Z)+y\right) \cap B_{R^{\prime}}(s) .
$$

From Remark 7] the diameter of the Voronoi cells of $P$ is less than $4 L R$, which is less than $R^{\prime}$. Hence,

$$
\pi_{j}(Y)=\pi_{j}(Z)+y
$$

We conclude with Lemma 5 and Proposition 6

\section{EXAmple of A PRIME Delone system}

In this section we give an example of a prime Delone system, i.e., a Delone system that does not have any nontrivial factor. The notion of prime topological dynamical systems was first introduced for $\mathbb{Z}$-actions in [FKS]. In [KN] a family of prime $\mathbb{R}$-actions was defined. Using a result in $\mathrm{Ad}$ this will provide the example we are looking for.

Let $(\alpha, \beta) \in(0,1)^{2}$. The circle sequence corresponding to the parameters $(\alpha, \beta)$ is the sequence $x=\left(x_{n}\right)_{n \in \mathbb{Z}}$ defined by $u_{n}=1$ if $\{n \alpha\}$ belongs to $[0, \beta)$ and 0 otherwise. Let $X_{\alpha, \beta}$ be the orbit closure of $x$ with respect to the shift map $T:\{0,1\}^{\mathbb{Z}} \rightarrow\{0,1\}^{\mathbb{Z}},\left(x_{n}\right)_{n} \mapsto\left(x_{n+1}\right)_{n}$, where $\{0,1\}^{\mathbb{Z}}$ is endowed with the product 
topology of the discrete topologies. Then $\left(X_{\alpha, \beta}, T\right)$ is a minimal subshift. We call it the circle subshift corresponding to the parameters $(\alpha, \beta)$ (it is called a circle map subshift in $[\mathrm{AD}$ ).

In $\mathrm{AD}$ the authors characterized circle subshifts that are linearly recurrent using a continued fraction algorithm, and in $\mathrm{Ad}$ the author shows that a circle sequence corresponding to the parameters $(\alpha, \beta)$ is a primitive substitutive sequence if and only if $\alpha$ and $\beta$ belong to the same quadratic field. We recall that primitive substitutive sequences are known to be linearly recurrent (see [DHS]).

Now consider the function $\left.g: X_{\alpha, \beta} \rightarrow\left\{a_{0}, a_{1}\right\} \subset\right] 0,+\infty\left[\right.$ defined by $g(x)=a_{x_{0}}$ such that $a_{0} / a_{1}$ does not belong to $\left.\left\{k_{1}+n \alpha\right) /\left(k_{2}+n \alpha\right): k_{1}, k_{2}, n \in \mathbb{Z}\right\}$. Let us construct the suspension of $\left(X_{\alpha, \beta}, T\right)$ with respect to $g$. Set

$$
Y_{\alpha, \beta}^{\prime}=\left\{(x, t): x \in X_{\alpha, \beta}, 0 \leq t \leq g(x)\right\}
$$

and let $Y_{\alpha, \beta}$ be the space obtained from $Y_{\alpha, \beta}^{\prime}$ by identifying pairs $(x, g(x))$ and $(T(x), 0)$. We define $\mathcal{S}_{\alpha, \beta}$, the $\mathbb{R}$-action on $Y_{\alpha, \beta}$, by:

$$
((x, s), t)=\left(T^{n} x, s+t-g_{n}(x)\right) \text { if }-s+g_{n}(x) \leq t<-s+g_{n+1}(x),
$$

where $g_{n}(x)=\sum_{k=0}^{n-1} g\left(T^{k} x\right)$ for $n>0, g_{n}(x)=-\sum_{k=-1}^{-n} g\left(T^{k} x\right)$ if $n<0$ and $g_{0}(x)=0$.

In $[\mathrm{KN}]$ it is proven that this $\mathbb{R}$-action is prime: it does not have any nontrivial factor whenever $\beta$ does not belong to $\alpha \mathbb{Z}$.

Now take $\alpha$ to be the golden mean, $\beta=\alpha+1, a_{0}=1$ and $a_{1}=\sqrt{2}$. Then, $\left(X_{\alpha, \beta}, T\right)$ is linearly recurrent and $\left(Y_{\alpha, \beta}, \mathcal{S}_{\alpha, \beta}\right)$ is prime.

It remains to show that $\left(Y_{\alpha, \beta}, \mathcal{S}_{\alpha, \beta}\right)$ is topologically conjugate to a linearly repetitive Delone system. Let $(x, t) \in Y_{\alpha, \beta}$ and set

$$
D(x, t)=\left(\left\{\sum_{k=0}^{n-1} g\left(T^{k} x\right): n \geq 0\right\} \bigcup\left\{-\sum_{k=-1}^{-n} g\left(T^{k} x\right): n \leq-1\right\}\right)-t
$$

(it is well-defined). It can be checked that this set is a linearly repetitive Delone set and $\left(D\left(Y_{\alpha, \beta}\right), \mathbb{R}\right)$ is a linearly repetitive Delone system. To conclude, it suffices to see that $D$ induces a topological conjugacy from $\left(Y_{\alpha, \beta}, \mathcal{S}_{\alpha, \beta}\right)$ to $\left(D\left(Y_{\alpha, \beta}\right), \mathbb{R}\right)$.

\section{ACKNOWLEDGMENTS}

The first author is grateful for the grant FONDECYT de Iniciación 11060002. She also thanks the Laboratoire Amiénois de Mathématique Fondamentale et Appliquée, CNRS-UMR 6140, Université de Picardie Jules Verne, where part of this work was done. The second author would like to acknowledge the hospitality of the Departamento de Matemática y Ciencia de la Computación de la Universidad de Santiago de Chile. The first and second authors were partially supported by Nucleus Millenius P04-069-F. All three authors acknowledge the financial support of program CNRS/CONICYT 2008 No. 21202.

\section{REFERENCES}

[Ad] B. Adamczewski, Codages de rotations et phénomènes d'autosimilarité, J. Th. Nombres de Bordeaux 14 (2002), 351-386. MR2040682 (2005b:37013)

[AD] B. Adamczewski, D. Damanik, Linearly recurrent circle map subshifts and an application to Schrödinger operators, Ann. Henri Poincaré 3 (2002), 1019-1047. MR1937613 (2004c:37017) 
[Du1] F. Durand, Linearly recurrent subshifts have a finite number of nonperiodic subshift factors, Ergod. Th. and Dynam. Sys. 20 (2000), 1061-1078. MR.1779393 (2001m:37022)

[Du2] F. Durand, Corrigendum and addendum to: Linearly recurrent subshifts have a finite number of nonperiodic subshift factors, Ergod. Th. and Dynam. Sys. 23 (2003), 663-669. MR:1972245 (2004c:37021)

[DHS] F. Durand, B. Host, C. Skau, Substitutional dynamical systems, Bratteli diagrams and dimension groups, Ergod. Th. and Dynam. Sys. 19 (1999), 953-993. MR:1709427 (2000i:46062)

[FKS] H. Furstenberg, H. B. Keynes, L. Shapiro, Prime flows in topological dynamics, Israel J. of Math. 14 (1973), 26-38. MR 0321055 (47:9588)

[HRS] C. Holton, C. Radin, L. Sadun, Conjugacies for tiling dynamical systems, Comm. Math. Phys. 254 (2005), 343-359. MR2117629 (2006m:37021)

[KN] H. B. Keynes, D. Newton, Real prime flows, Trans. Amer. Math. Soc. 217 (1976), 237-255. MR0400189 (53:4024)

[LP] J. C. Lagarias, P. A. B. Pleasants, Repetitive Delone sets and quasicrystals, Ergod. Th. and Dynam. Sys. 23 (2003), 831-867. MR.1992666 (2005a:52018)

[Pe] K. Petersen, Factor maps between tiling dynamical systems, Forum Math. 11 (1999), 503512. MR1699171 (2000f:37019)

[RS] C. Radin, L. Sadun, Isomorphism of hierarchical structures, Ergod. Th. and Dynam. Sys. 21 (2001), 1239-1248. MR1849608 (2002e:37021)

[Ro] E. A. Robinson, Jr., Symbolic dynamics and tilings of $\mathbb{R}^{d}$, Symbolic dynamics and its applications, 81-119, Proc. Sympos. Appl. Math., 60, Amer. Math. Soc., Providence, RI, 2004. MR2078847(2005h:37036)

[Ru] D. J. Rudolph, Markov tilings of $\mathbb{R}^{n}$ and representations of $\mathbb{R}^{n}$ actions, Measure and measurable dynamics (Rochester, NY, 1987), 271-290, Contemp. Math., 94, Amer. Math. Soc., Providence, RI, 1989. MR1012996 (91b:28016)

[So1] B. Solomyak, Dynamics of self-similar tilings, Ergod. Th. and Dynam. Sys. 17 (1997), 695-738. MR 1452190 (98f:52030)

[So2] B. Solomyak, Nonperiodicity implies unique composition for self-similar translationally finite tilings, Discrete Comput. Geom. 20 (1998), 265-279. MR 1637896 (99f:52028)

[So3] B. Solomyak, Spectrum of dynamical systems arising from Delone sets, In: Quasicrystals and discrete geometry, Amer. Math. Soc., Providence, RI, 1998, 265-275. MR 1636783 (99f:58176)

Departamento de Matemática y Ciencia de la Computación, Universidad de Santiago de Chile, Avenida libertador Bernardo O'Higgins 3363, Santiago, Chile

E-mail address: maria.cortez@usach.cl

Laboratoire Amiénois de Mathématique Fondamentale et Appliquée, CNRS-UmR 6140, Université de Picardie Jules Verne, 33 rue Saint Leu, 80039 Amiens Cedex, France

E-mail address: fabien.durand@u-picardie.fr

Laboratoire Amiénois de Mathématique Fondamentale et Appliquée, CNRS-UmR 6140, Université de Picardie Jules Verne, 33 rue Saint Leu, 80039 Amiens Cedex, France

E-mail address: samuel.petite@u-picardie.fr 